of -1.4 atmospheres. Naturally, the value for $\Delta P^{\prime}$ calculated using the formula for the change in vapor pressure with applied pressure,

$$
d P / d P^{\prime}=V^{\prime} / V
$$

(where $P$ is the equilibrium pressure above a liquid on which a pressure $P^{\prime}$ is exerted, while $V$ and $V^{\prime}$ are, respectively, the molar volumes of vapor and liquid), is also -1.40 atmospheres. This is obvious, since Eq. (4) is the two-dimensional analog of Eq. (6). Both of these values are in excellent agreement with the value of -1.3 atmospheres calculated from the shift in lambda-point of bulk helium. The inflection in the isotherm appears, therefore, as a result of a rather abrupt change in surface density on going from the second to the third layer as calculated by means of the lambdapoint shift.

This last result is given as evidence for the contention that the first few layers tend to complete themselves before starting to build new layers. It assumes that the effective density of the film is equal to that of the completed films. This means that the film consists largely of regions of the surface completely filled as predicted by Halsey. ${ }^{18}$

\section{ACKNOWLEDGMENT}

The authors are indebted to Mr. R. J. Tykodi for his collaboration in the determination of the heat capacities of helium adsorbed on the high surface area $\mathrm{TiO}_{2}$.

${ }^{18}$ G. Halsey, J. Chem. Phys. 16, 931 (1948).

\title{
I. Normal Frequencies of a One-Dimensional Crystal. II. An Approximation to the Lattice Frequency Distribution in Isotropic Solids
}

\author{
J. O. HALFORD \\ Chemistry Department, University of Michigan, Ann Arbor, Michigan
}

(Received March 19, 1951)

\begin{abstract}
I. The accurate expression for the individual frequencies of a linear crystal of any number of particles is derived.

II. It is shown that, for an isotropic three-dimensional array of uniform masses, the root-mean-square frequency is easily evaluated and the maximum square must always be less, but not much less, than twice the mean square. For two commonly studied simple cubic systems, the maximum square is evaluated and shown to be twice the mean square. The Debye expression for the frequencies in terms of standing wave components is modified empirically to give the correct maximum and mean squares by substituting linear crystal frequencies for the components and introducing a second force constant.

The resulting expression, a simplified form of the factored secular equation, should yield a more realistic and probably therefore a more widely useful distribution function than the Debye equation.
\end{abstract}

I.

$I^{1}$ $\mathrm{N}$ connection with the normal frequency distribution in solids, it has proved to be useful and instructive to examine the frequencies of a so-called one-dimensional crystal. This has been done by Born and von Kármán ${ }^{1}$ and is discussed by Mayer and Mayer. ${ }^{2}$ For $N$ point masses $m$ with a restoring force $k$ for unit relative displacement of neighbors, the $n$th normal frequency is given by the equation

$$
\nu_{n}=\left(k / \pi^{2} m\right)^{\frac{1}{2}} \sin [n \pi / 2(N-1)] ;(1<n<N-2) .
$$

Since the equation expresses $N-2$ frequencies for a model which must have $N-1$ vibrations, the result is an approximation which is claimed to approach accuracy only for large values of $N$.

The accurate solution, with this restriction removed, is readily obtained. As might be expected, the correction, for large $N$, is negligible; but the effect of the

\footnotetext{
${ }^{1}$ M. Born and Th. von Kármán, Physik. Z. 13, 297 (1912).

2 J. E. Mayer and M. G. Mayer, Statistical Mechanics (John Wiley and Sons, Inc., Nẹw York, 1946), p. 248.
}

approximation is exactly evaluated, and the new result is valid for all $N$ down to the diatomic molecule and may find application in future studies of limited chains of particles.

For the model, as described, the kinetic energy is $m \sum \dot{X}_{i}{ }^{2} / 2$ and the potential energy is $k \sum\left(X_{i}-X_{i-1}\right)^{2} / 2$. With the substitution, $u\left(=4 \pi^{2} \nu^{2} m / k\right)-2$, the secular equation takes the form

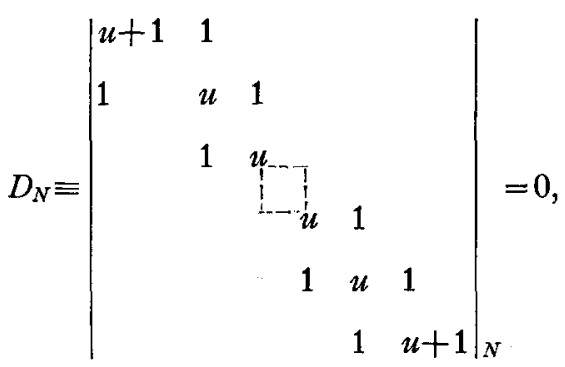

with $N$ denoting the number of rows or columns in the determinant, 
The translation, $u+2=0$, can be factored_out. Let $E_{N}$ be defined as

$$
E_{N}=\left|\begin{array}{llllll}
u & 1 & & & & \\
1 & u & 1 & & & \\
& 1 & u & & \\
& & 1 & u & 1 & \\
& & & 1 & u & 1 \\
& & & & 1 & u
\end{array}\right|_{N}
$$

Then, by means of expansion by minors, we obtain

$$
\begin{aligned}
D_{N} & =(u+1)^{2} E_{N-2}-2(u+1) E_{N-3}+E_{N-4} \\
& =\left(u^{2}+2 u\right) E_{N-2}-(u+2) E_{N-3}=(u+2) E_{N-1} .
\end{aligned}
$$

From Eq. (4) it is seen that the normal frequencies for the $N$-particle chain are the roots of the equation $E_{N-1}=0$. To factor this equation it is assumed that Eq. (1) has the correct form except that the angle may be wrong. The frequency is then taken to be $\left(k / \pi^{2} m\right)$ $\sin \theta / 2$, so that $\left(4 \pi^{2} \nu^{2} m / k\right)-2=-2\left(1-2 \sin ^{2} \theta / 2\right)$, leading to the substitution $u=-2 \cos \theta$.

For $N=2, \quad E_{1}=-2 \cos \theta=-\sin 2 \theta / \sin \theta$, and, for $N=3, E_{2}=4 \cos ^{2} \theta-1=\sin 3 \theta / \sin \theta$. Now, through expansion by minors,

$$
\begin{aligned}
\sin \theta E_{3} & =-2 \cos \theta \sin 3 \theta+\sin 2 \theta \\
& =-2 \cos \theta \sin 3 \theta+\sin 3 \theta \cos \theta-\cos 3 \theta \sin \theta \\
& =-\sin 3 \theta \cos \theta-\cos 3 \theta \sin \theta=-\sin 4 \theta
\end{aligned}
$$

and

$$
\begin{aligned}
\sin \theta E_{4} & =2 \cos \theta \sin 4 \theta-\sin 3 \theta \\
& =2 \cos \theta \sin 4 \theta-\sin 4 \theta \cos \theta+\cos 4 \theta \sin \theta \\
& =\sin 4 \theta \cos \theta+\cos 4 \theta \sin \theta=\sin 5 \theta .
\end{aligned}
$$

Since this operation can be extended indefinitely with increasing values of $N$, the general secular equation for the internal motions takes the form, for $N$ particles,

$$
E_{N-1}=(-1)^{N-1} \sin N \theta / \sin \theta=0 .
$$

As an alternative, it is readily shown that if $E_{N-1}$ $= \pm \sin N \theta / \sin \theta$ and $E_{N}=\mp \sin (N+1) \theta / \sin \theta$, then $E_{N+1}$ $= \pm \sin (N+2) \theta / \sin \theta$, which, in conjunction with the known values of $E_{1}$ and $E_{2}$, leads to the same conclusion.

Equation (6) is satisfied when $\sin N \theta=0$ and $\sin \theta \neq 0$, for which the condition is that $\theta=n \pi / N$, where $n$ must not be zero nor a multiple of $N$. Although the number of such angles is infinite, they yield only $N-1$ values of $u$, which lie between -2 and +2 and are all encountered in the range $0<\theta<\pi$, for which $1<n<N-1$. Since the secular equation $E_{N-1}=0$ can have only $N-1$ roots, there are no other values of $u$, real or imaginary, which will satisfy it. It follows from the definition of $\theta$ that the $n$th frequency is

$$
\nu_{n}=\left(k / \pi^{2} m\right)^{\frac{1}{2}} \sin (n \pi / 2 N) \text {. }
$$

Evidently, the approximation involved in deriving Eq. (1), namely, the arbitrary restriction of the terminal masses to zero amplitude, has given an equation for the $N$-particle chain which is actually correct for $N-1$ particles. In the same way, if, instead of factoring out the translation, the end corrections of Eq. (2) had been neglected by replacing each element $u+1$ by $u$, the resulting frequency distribution would be correct for $N+1$ instead of $N$ particles. Since, for $N$ sufficiently large, the distribution function is independent of the chain length, it is evident that under this condition the end corrections are negligible. It is reasonable, then, to assume that they will also be negligible in two- and three-dimensional systems.

In deriving Eq. (7), no end corrections have been neglected, nor have any approximations other than those implied in the potential energy function been introduced. Consequently, Eq. (7) must yield correctly the frequency of a symmetrical diatomic molecule, for which $n=1$ and $N=2$. The frequency becomes $\nu=(k /$ $\left.\pi^{2} m\right)^{\frac{1}{2}} \sin \pi / 4$ or $(1 / 2 \pi)(2 k / m)^{\frac{1}{4}}$, which is the familiar expression for this case. By Eq. (1), as well as by inspection, this is also the frequency for a single mass $m$ connected to two fixed points by bonds of force constant $k$.

\section{II.}

In solving for the distribution of lattice frequencies in a solid, it is necessary to work with a particular model involving a defined distribution of atoms and an assumed potential energy function. The formidable features of the derivation may lead to approximations which widen the gap between the model and physical reality.

When the problem has been solved for a particular model, it is of interest to ask if the result is applicable to other models as well, that is, if the distributions of a representative set of models are similar enough to permit the substitution of one for another. To obtain an adequate number of derived distributions to resolve this question appears at present to be too difficult, but the general usefulness of a particular distribution can be judged by the number and variety of solid substances for which the thermal properties can be calculated successfully.

Frequency distributions, or partial descriptions of the distribution, have been proposed by Debye, ${ }^{3}$ Born and von Kármán, ${ }^{1}$ Blackman, ${ }^{4}$ Houston, ${ }^{5}$ Leighton, ${ }^{6}$ Montroll, ${ }^{7}$ and others. Of these, the relatively simple Debye description has been most widely tested and used. The Debye theory stands out from the others because of the highly simplified model, an isotropic continuum which can represent reality only in the limit for very low frequencies. Since the Debye distribution cannot

\footnotetext{
${ }^{3}$ P. P. Debye, Ann. Physik 39, 789 (1912).

4 M. Blackman, Proc. Roy. Soc. (London) A159, 416 (1937).

${ }^{5}$ W. V. Houston, Revs. Modern Phys. 20, 161 (1948).

6 R. B. Leighton, Revs. Modern Phys. 20, 165 (1948).

7 E. W. Montroll, J. Chem. Phys. 15, 575 (1947).
} 
be accurate in the higher frequency range, the use of his equations to predict thermal properties at higher temperatures must be regarded as empirical. Each of the other distributions is likewise a property of a particular model. Most of the available distributions are incomplete because of difficulties of computation, but the application of a complete accurate one to the prediction of thermal properties of other models would, like the Debye theory, have to be made upon an empirical basis.

It would seem, therefore, that, for success in predicting thermal properties, it makes little difference whether the distribution is merely set down empirically or is arrived at in some more elegant and complicated fashion. In either case, a proposal that the function is generally usable must be validated empirically.

That the frequency distribution, for the calculation of thermal properties, does not have to be accurate throughout the range is evident from the success of the Debye theory in predicting the entire heat capacity curve for some simple crystals with fair accuracy in spite of the unreal model upon which the theory is based.

It is further evident, from the Einstein function, the Nernst-Lindemann equation, and the recent calculations of $\operatorname{Raman}^{8}$ and his collaborators, that considerable success is possible even with a severely oversimplified function. The sensitivity of the thermal properties to the frequency distribution has been analyzed in detail by Katz. ${ }^{9}$

The present purpose is to modify the Debye distribution empirically in the direction indicated by the study of more realistic models than the Debye continuum. To effect an improvement, it is necessary only to make changes in the right directions and clearly unnecessary to include specific features of any particular distribution, 'such as, for example, the "infinities" of the Montroll function.

For a cubic Debye model, three sets of standing waves which can be set up parallel to the edges of the cube are recognized. The propagation vector of a characteristic standing wave of the model is taken to be the sum of three component propagation vectors, one taken from each of the sets parallel to the edges. The components are combined in all possible ways. Since the propagation vector is proportional to the frequency, the characteristic frequencies can be expressed by the vector summation

$$
\nu^{2}=\nu_{x}^{2}+\nu_{y}{ }^{2}+\nu_{z}^{2},
$$

in which $\nu_{x}$, etc., are the frequencies of the component standing waves. Equation (8) defines spherical surfaces of constant frequency and sets the number of frequencies between $\nu$ and $\nu+d \nu$ proportional to the volume of a spherical shell of radius $\nu$. If this proportionality is assumed to persist all the way to an

${ }^{8}$ C. V. Raman and others, J. Indian Acad. Sci. A14 (1941); $A 15$ (1942).

${ }^{9}$ E. Katz, J. Chem. Phys. 19, 488 (1951). arbitrarily imposed maximum frequency $\nu_{0}$, the distribution function $z$ becomes

$$
z=9 N \nu^{2} d \nu / \nu_{0}^{3},
$$

which is the original Debye relation, used subsequently without change in most published discussions.

In this form, however, the function does not properly represent the model. The assumption of a maximum frequency requires, for the vector additions, that each component be limited to a maximum value. All of the vectors of Eq. (8) must then terminate within a cube and the number of frequencies in the interval $\nu$ to $\nu+d \nu$ will be proportional to the part of the area of a spherical shell which lies within the cube. Equation (9) can be valid only for frequencies less than the maximum component. The distribution function should contain two discontinuities, at $\left(\frac{1}{3}\right)^{\frac{3}{3}} \nu_{0}$ and $\left(\frac{2}{3}\right)^{\frac{1}{1}} \nu_{0}$, with the function diminishing to zero at the maximum frequency. These features have been discussed in detail by Slater, ${ }^{10}$ along with related interpretations. It is surprising that they have been rather generally ignored in publications of later date than Slater's discussion.

The use of the simple Debye distribution over the whole range sets the mean frequency too high by neglecting the discontinuities and consequently including a large number of extraneous high frequencies. The well-known success of the function in certain cases must then mean that at least one compensating factor has been included which tends to lower the mean frequency. The nature of this second factor can be inferred from a comparison of the linear crystal distribution with that of a continuous rod. In the continuous one-dimensional model, the standing wave frequencies are equally spaced, with the numerical mean at half the maximum and the root mean square at $\nu(\max ) / \sqrt{3}$. In the linear crystal, by Eq. (7), for each frequency proportional to $\sin (n \pi / 2 N)$ there is always another proportional to $\sin [(N-n) \pi / 2 N]$ or $\cos (n \pi / 2 N)$, unless $n=N / 2$, which is trivial. The sum of the squares of these two frequencies is the square of the maximum frequency, the mean square is half the maximum square, and the root mean square is $\nu(\max ) / \sqrt{2}$, showing that the mean frequency of the continuous model is too low. This property will certainly appear in the continuous two- or three-dimensional model, at least to some degree, and will account qualitatively for a cancellation of inadequacies in the Debye distribution in favorable cases.

In deference to the considerable success of the Debye equation, it seems to be in order to retain the form of Eq. (8), but to attempt to find a set of components $\nu_{x}$, etc., which will give a more realistic mean frequency. Equation (8) represents a factored form of the secular equation which is undoubtedly severely oversimplified, since in certain cases the secular equation has been reduced to a cubic in $\nu^{2}$ which is not readily factored

${ }^{10} \mathrm{~J}$. C. Slater, Introduction to Chemical Physics (McGraw-Hill Book Company, Inc., New York, 1939), pp. 225 ff. 
in any general way to give the individual frequencies explicitly. It could still be true, however, that Eq. (8) with the right set of components would give a distribution which could be used for more than one model.

At this point, it will be useful to examine the secular equation for an isotropic array of uniform masses. In any such array, if end corrections are neglected, each particle is symmetrically surrounded by neighbors, and the restoring force for unit displacement of a single particle when the entire model is motionless will be independent of the direction of the displacement. With any specified potential energy function and any chosen cartesian coordinates, the secular determinant must contain the unknown squared frequency in a uniform element which appears at every point on the principal diagonal. For any reasonable potential energy function, it should always be easy to express this diagonal element. All other elements of the determinant will be potential constants. If every element is divided by the principal force constant $k$, the diagonal element will be $u=\left(4 \pi^{2} v^{2} m / k\right)-A$, in which $A$ is a dimensionless quantity made up of the sum of ratios of potential constants. The secular equation has the degree $n=3 N$ for $N$ particles and the first term in the polynomial form is $u^{n}$. There can be no term in $u^{n-1}$, however, since in a term by term expansion it is not permissible to accept at any time two elements from the same row or column and the selection of $u,(n-1)$ times, blocks out all elements except the remaining $u$.

As a consequence the sum of the roots $u_{i}$ is zero, the mean value of $\lambda_{i}{ }^{\prime}\left(4 \pi^{2} \nu_{i}^{2} m / k\right)$ is $A$ and the average squared frequency is $k A / 4 \pi^{2} m$. This evidently gives the frequency with which a single particle would vibrate if the rest of the model was motionless.

There are some models in which it is possible to vibrate all the particles simultaneously in this same manner against the same set of forces. If the displacement has a component directed toward a neighbor, the neighbor will have a like component directed back toward the particle. If, however, there is no component toward a particular neighbor, the two can move in the same direction. Evidently, all the relative displacements are twice as large as they were when the particle was moving alone, and the restoring force must also have been doubled for unit displacement of the particle. Consequently, the squared frequency of the general motion is twice that of the individual particle which has been evaluated from the diagonal element. For such models, then, the squared maximum frequency is twice the numerical mean square.

Two simple cubic models which have been studied extensively fulfill this condition. In Houston's ${ }^{5}$ model, a unit relative displacement of neighbors along their line of centers is resisted by a force $k$, while a displacement perpendicular to the connecting line sets up a force $k \alpha$, where $\alpha$ is a fraction. The two squared frequencies, by inspection, are $\lambda^{\prime}($ mean $)=2+4 \alpha$ and $\lambda^{\prime}(\max )=4+8 \alpha$. Each row of particles, at the maximum frequency, executes the highest frequency motion of the linear crystal, but any two neighboring rows are always out of phase. For the central force model introduced by Born and von Kármán ${ }^{1}$ and used by Black$\operatorname{man}^{4}$ and Montroll, ${ }^{7}$ a restoring force $k$ is assigned to the unit displacement toward a nearest neighbor, and a force $k \alpha$ to the displacement toward a second neighbor across the diagonal of a square. Here again, by inspection, the squared frequencies are $\lambda^{\prime}($ mean $)=2+4 \alpha$ and $\lambda^{\prime}(\max )=4+8 \alpha$; but, for motion parallel to an edge of the cubical model, while each row again executes the motion of maximum frequency of the linear crystal, the rows are now all in phase when the frequency is at the maximum.

If only forces between nearest neighbors were important, it would be generally true that the mean square is half the maximum square, and it should always be true that the contribution of the principal force constant to the mean square frequency is half its contribution to the maximum square in an isotropic array. Every force constant must contribute to the mean square, but there will be second and more distant neighbors in some models which suffer no relative displacement in the highest frequency motion. This can be seen by examining the linear crystal with an additional force $k \alpha$ introduced for the relative displacement of second neighbors, resulting in $\lambda^{\prime}($ mean $)=2+2 \alpha$ and $\lambda^{\prime}(\max )=4$. It is concluded, for isotropic arrays, that the mean square frequency is at least half the maximum square and may be larger, but not much larger.

With a reasonable potential energy function, it will always be possible to write the diagonal element of the secular determinant which evaluates the root mean square frequency, and it will usually also be possible to find the maximum frequency. An acceptable distribution function and simplified secular equation should express these two frequencies with reasonable accuracy in a nearly correct ratio.

In the first approximation the maximum square is twice the mean square. A wide variety of distributions will meet this condition, from something like the linear crystal distribution at one extreme to a heavy concentration about two principal frequencies at the other. The latter extreme is suggested by Montroll's" "infinities," but the former, intuitively, seems more in line with the widely variable situations presumably represented by the normal coordinates. It is possible that the distributions for real systems may vary all the way from one extreme to the other, and still possible also that the representation of thermal properties may not be sensitive to the particular distribution chosen within the prescribed limits.

The limiting value of the required ratio can be imposed upon the Debye distribution by interpreting the components of Eq. (8) as linear crystal frequencies, according to Eq. (7). Since the one-dimensional frequencies are arranged in complementary pairs which meet the requirement, it is obvious that the sums can 
be arranged as similar complementary pairs. At the same time the expected discontinuities appear in the distribution. The vector additions still outline a cube whose geometry requires the discontinuities, but the density of frequencies within the cube is no longer constant, but instead varies with the coordinates. This change alone should effect an improvement in the Debye distribution by introducing some features required of a real system, and the resulting distribution might very well fit the thermal properties of a wider selection of models without further modification.

In this form, however, Eq. (8) as an approximation to the secular equation is too highly simplified, since it implies a potential energy function with only the one constant introduced through Eq. (7), and no one would attempt to describe a reasonable approximation to a real system without at least two constants. A further modification is therefore suggested, in order to bring Eq. (8) into line with the common maximum frequency of the Houston ${ }^{5}$ and central force models of the simple cubic system without altering the ratio of the maximum to the mean square. This can be done by rewriting Eq. (8) in the form

$$
\nu^{2}=\nu_{x}^{2}+\alpha\left(\nu_{y}{ }^{2}+\nu_{z}^{2}\right)
$$

with the components still taken from Eq. (7).

Equation (9) is still an oversimplified secular equation in respect to real systems and presumably also most mathematical models, but it should now be markedly better than the Debye equation and should be usable to express the thermal properties of a wide variety of isotropic models adequately. The equation implies a potential energy function, and therefore a model, without specifying what they are. There are preliminary indications that Eq. (9) is the exact form of the factored secular equation for Houston's ${ }^{5}$ model; but the proof requires further development, and, if achieved, will be reported at a later date.

To obtain all the normal frequencies, Eq. (9) must be applied three times, once with each set of components in the leading term. For a cube of $N^{3}$ particles, there will be $N^{3}$ vector additions each time, leading to the correct total of $3 N^{3}$ frequencies. The first set of frequencies is obtained by adding the vectors $\nu_{x}, \alpha^{\frac{1}{1}} \nu_{y}$ and $\alpha^{\frac{1}{2}} \nu_{z}$. All of the vectors must terminate within a rectangular parallelipiped of square cross section, a construction which will introduce four discontinuities into the distribution curve, at $\alpha^{\frac{1}{t}} \nu_{x}(\max ),(2 \alpha)^{\frac{1}{2}} \nu_{x}(\max )$, $\nu_{x}(\max )$ and $(1+\alpha)^{\frac{1}{2}} \nu_{x}(\max )$. The density of frequencies within the parallelipiped increases with the distance from each of the coordinate reference planes. Surfaces of constant frequency are still spherical, with the pertinent surface area increasing rapidly up to the first discontinuity, more slowly from the first to the second, than decreasing slightly from the second to the third, decreasing more sharply between the third and the fourth, and dropping rapidly to zero between the fourth discontinuity and the maximum frequency. Such a distribution has more acute discontinuities than the Debye distribution, but will not show the extreme "infinities" indicated by Montroll's analysis. The result deviates from the Debye distribution in the direction of the Montroll type of curve and should be an improvement over the Debye function which is probably about as near as one can expect to come to a general function for isotropic crystals.

At very low frequencies, and therefore for thermal properties at very low temperatures, with the components $\nu_{x}$, etc., in the region where $\theta=\sin \theta$, it is evident from Eq. (7) that the distribution follows the Debye quadratic form. How low the temperature must be, however, depends upon the value of $\alpha$ which, if small enough, would lead to a linear crystal distribution in the limit. Such a distribution appears at present to be extremely unlikely for any real system. Consequently, no change in the commonly used method of extrapolating low temperature thermal data has been suggested. 